\title{
Morse indices and the number of maximum points of some solutions to a two-dimensional elliptic problem
}

\author{
Futoshi Takahashi
}

\begin{tabular}{|c|l|}
\hline Citation & OCAMI Preprint Series \\
\hline Issue Date & 2008 \\
\hline Type & Preprint \\
\hline Textversion & Author \\
\hline Rights & For personal use only. No other uses without permission. \\
\hline Relation & $\begin{array}{l}\text { This is a pre-print of an article published in Archiv der } \\
\text { Mathematik. The final authenticated version is available online } \\
\text { at: } \underline{\text { https://doi.org/10.1007/s00013-009-0021-8 }} .\end{array}$ \\
\hline
\end{tabular}

From: Osaka City University Advanced Mathematical Institute http://www.sci.osaka-cu.ac.jp/OCAMI/publication/preprint/preprint.html 


\title{
Morse indices and the number of maximum points of some solutions to a two-dimensional elliptic problem
}

\author{
Futoshi Takahashi \\ Department of Mathematics, Osaka City University \\ Sumiyoshi-ku, Osaka, 558-8585, Japan \\ Tel: $(+81)(0) 6-6605-2508$ \\ E-mail: futoshi@sci.osaka-cu.ac.jp
}

Abstract. In this note, we consider the problem

$$
-\Delta u=u^{p} \quad \text { in } \Omega, \quad u>0 \quad \text { in } \Omega,\left.\quad u\right|_{\partial \Omega}=0
$$

on a smooth bounded domain $\Omega$ in $\mathbb{R}^{2}$ for $p>1$. Let $u_{p}$ be a positive solution of the above problem with Morse index less than or equal to $m \in \mathbb{N}$. We prove that if $u_{p}$ further satisfies the assumption $p \int_{\Omega}\left|\nabla u_{p}\right|^{2} d x=O(1)$ as $p \rightarrow \infty$, then the number of maximum points of $u_{p}$ is less than or equal to $m$ for $p$ sufficiently large. If $\Omega$ is convex, we also show that a solution of Morse index one satisfying the above assumption has a unique critical point and the level sets are star-shaped for $p$ sufficiently large.

Keywords: Morse index, maximum point, semilinear elliptic equation.

2000 Mathematics Subject Classifications: 35B40, 35J20, 35J25.

\section{Introduction.}

In this note we consider the problem

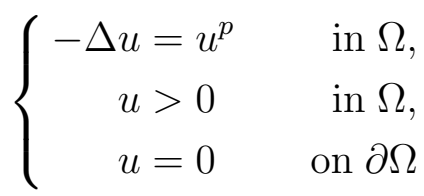

where $\Omega$ is a smooth bounded domain in $\mathbb{R}^{2}$ and $p>1$. Since the Sobolev embedding $H_{0}^{1}(\Omega) \hookrightarrow L^{p+1}(\Omega)$ is compact for any $p>1$, the existence of at least one solution is easy to obtain. In fact, if we define

$$
S_{p}=\inf _{u \in H_{0}^{1}(\Omega)}\left\{\left.\int_{\Omega}|\nabla u|^{2} d x\left|\int_{\Omega}\right| u\right|^{p+1} d x=1\right\}
$$


then a standard variational method implies that $S_{p}$ is achieved by a positive function $\bar{u}_{p} \in H_{0}^{1}(\Omega)$ and $u_{p}=S_{p}^{1 /(p-1)} \bar{u}_{p}$ is a solution of (1.1).

For the least energy solution $u_{p}$ obtained in this way, several studies on the asymptotic behavior have been done in [6], [7], [3] and [1]. In particular, in [6] and [7], along a suitable subsequence $p \rightarrow \infty$, least energy solution $u_{p}$ is shown to develop a single spiky pattern at an interior point of the domain. More precisely, $u_{p}$ satisfies a uniform $L^{\infty}$-norm estimate

$$
C_{1} \leq\left\|u_{p}\right\|_{L^{\infty}(\Omega)} \leq C_{2}
$$

for some $0<C_{1} \leq C_{2}<\infty$ independent of $p$, and to "concentrate" at an interior point of the domain, i.e.,

$$
\frac{u_{p}^{p}}{\int_{\Omega} u_{p}^{p} d x} \rightarrow \delta\left(x_{0}\right) \quad \text { as } p \rightarrow \infty
$$

for some $x_{0} \in \Omega$ in the sense of Radon measures. Moreover, the estimate

$$
p \int_{\Omega}\left|\nabla u_{p}\right|^{2} d x \rightarrow 8 \pi e \quad \text { as } p \rightarrow \infty
$$

is proved for least energy solution $u_{p}$. Recently, for any $m \in \mathbb{N}$, a solution sequence $\left\{u_{p}\right\}$ which exhibits the asymptotic behavior

$$
p \int_{\Omega}\left|\nabla u_{p}\right|^{2} d x \rightarrow 8 \pi m e \quad \text { as } p \rightarrow \infty
$$

has been constructed in [5] under some topological assumption of the domain.

In the following, we restrict our attention to the solution $u_{p}$ of (1.1) which satisfies the assumption

$$
p \int_{\Omega}\left|\nabla u_{p}\right|^{2} d x=O(1) \quad \text { as } p \rightarrow \infty .
$$

Before stating the results in this paper, we recall that the Morse index of a solution $u$ of (1.1) is the number of negative eigenvalues of the linearized operator $L_{u}=-\Delta-p u^{p-1}$. acting on $H_{0}^{1}(\Omega)$. In this paper, we prove the following theorems.

Theorem 1. Let $\left\{u_{p}\right\}$ be a solution sequence of (1.1) satisfying the assumption (1.2) with the Morse index less than or equal to $m, m \in \mathbb{N}$. Then the 
number of maximum points of $u_{p}$ is less than or equal to $m$ for $p$ sufficiently large.

Theorem 2. Let $\left\{u_{p}\right\}$ be a solution sequence of (1.1) satisfying the assumption (1.2) with the Morse index one. If $\Omega$ is convex, then $u_{p}$ has only one critical point $x_{p}$ which is the global maximum point of $u_{p}$, and

$$
\left(x-x_{p}\right) \cdot \nabla u_{p}(x)<0, \quad \forall x \in \Omega \backslash\left\{x_{p}\right\}
$$

holds for $p$ sufficiently large. In particular, the level sets of $u_{p}$ are strict star-shaped with respect to $x_{p}$.

In [4], El Mehdi and Pacella treated the problem

$$
\left\{\begin{aligned}
-\Delta u & =N(N-2) u^{p-\varepsilon}-\lambda u & & \text { in } \Omega, \\
u & >0 & & \text { in } \Omega, \\
u & =0 & & \text { on } \partial \Omega
\end{aligned}\right.
$$

where $\Omega$ is a smooth bounded, star-shaped domain in $\mathbb{R}^{N}, N \geq 3, p=$ $(N+2) /(N-2), \varepsilon>0$ and $\lambda \geq 0$. They proved similar results on the relation between the Morse index and the number of maximum points of blowing-up solutions $\left\{u_{\varepsilon}\right\}$ to this problem via a blow-up analysis. Note that in this case, it holds $\left\|u_{\varepsilon}\right\|_{L^{\infty}(\Omega)} \rightarrow+\infty$ as $\varepsilon \rightarrow 0$. Theorems in this paper are two-dimensional counterparts to the results in [4]. However, in our situation, solutions may not blow up in the $L^{\infty}$-norm sense, so the usual blowup analysis as in the higher-dimensional case does not work. To overcome this difficulty, we combine the arguments in [4] and the two-dimensional blow-up technique by Adimurthi and Grossi [1].

\section{Proof of Theorem 1.}

Let $x_{p}^{1} \in \Omega$ be a maximum point of $u_{p}$ for $p$ large, that is, $\left\|u_{p}\right\|_{\infty}=u_{p}\left(x_{p}^{1}\right)$. First, we recall a result in [6] that for any solution $u_{p}$ of (1.1), there holds an estimate

$$
\left\|u_{p}\right\|_{\infty} \geq \lambda_{1}^{1 /(p-1)}
$$

where $\lambda_{1}>0$ is the first eigenvalue of $-\Delta$ under the Dirichlet boundary condition. Proof of this fact in [6] is simple, so we recall it here for the readers' convenience. Let $\phi_{1}>0$ be the first eigenfunction associated to $\lambda_{1}$. 
Then we see

$$
0=\int_{\Omega}\left(u_{p} \Delta \phi_{1}-\phi_{1} \Delta u_{p}\right) d x=\int_{\Omega} \phi_{1} u_{p}\left(u_{p}^{p-1}-\lambda_{1}\right) d x,
$$

thus we have $\left\|u_{p}\right\|_{\infty}^{p-1} \geq \lambda_{1}$.

From this fact, we see

$$
\varepsilon_{p}:=\frac{1}{\sqrt{p}\left\|u_{p}\right\|_{\infty}^{(p-1) / 2}} \rightarrow 0 \quad(p \rightarrow \infty) .
$$

Next, we claim that $x_{p}^{1}$ is away from $\partial \Omega$ uniformly in $p$ sufficiently large. Indeed, (1.1) and (1.2) imply that $p \int_{\Omega} u_{p}^{p+1} d x=O(1)$ as $p \rightarrow \infty$. Then we have

$$
p \int_{\Omega} u_{p}^{p} d x \leq\left(p \int_{\Omega} u_{p}^{p+1} d x\right)^{p /(p+1)} p^{1 /(p+1)}|\Omega|^{1 /(p+1)} \leq C
$$

uniformly in $p$, thus $\int_{\Omega} u_{p}^{p} d x=O(1 / p)$ as $p \rightarrow \infty$. Therefore, at any maximum point $x_{p}$ of $u_{p}, v_{p}(x):=u_{p}(x) / \int_{\Omega} u_{p}^{p} d x$ satisfies

$$
v_{p}\left(x_{p}\right)=\frac{u_{p}\left(x_{p}\right)}{\int_{\Omega} u_{p}^{p} d x} \geq \frac{\lambda_{1}^{1 /(p-1)}}{\int_{\Omega} u_{p}^{p} d x} \rightarrow+\infty
$$

as $p \rightarrow \infty$. On the other hand, applying Lemma 4.1 in [6] and the elliptic $L^{1}$ estimate to $v_{p}$, we have, as in Lemma 4.2 in [6], that $v_{p}$ is bounded in $L^{\infty}$ near $\partial \Omega$ uniformly in $p$. Thus any maximum point $x_{p}$ of $u_{p}$ cannot approach to $\partial \Omega$ and for some neighborhood $\omega$ of $\partial \Omega$, we have $\left\{x_{p} \in \Omega \mid u_{p}\left(x_{p}\right)=\left\|u_{p}\right\|_{\infty}\right\} \subset$ $\Omega \backslash \omega$ for large $p$.

Now, we define the scaled function

$$
\tilde{u}_{p}(y)=\frac{p}{\left\|u_{p}\right\|_{\infty}}\left\{u_{p}\left(\varepsilon_{p} y+x_{p}^{1}\right)-u_{p}\left(x_{p}^{1}\right)\right\}, \quad y \in \Omega_{p}^{1}=\frac{\Omega-x_{p}^{1}}{\varepsilon_{p}}
$$

as in [1], which satisfies

$$
\left\{\begin{array}{lr}
-\Delta \tilde{u}_{p}(y)=\left(1+\frac{\tilde{u}_{p}}{p}(y)\right)^{p} & \text { in } \Omega_{p}^{1} \\
0<1+\frac{\tilde{u}_{p}}{p}(y) \leq 1 & \text { in } \Omega_{p}^{1} \\
\tilde{u}_{p}(y)=-p & y \in \partial \Omega_{p}^{1}
\end{array}\right.
$$


By the above claim, the limit domain of $\Omega_{p}^{1}$ as $p \rightarrow \infty$ is $\mathbb{R}^{2}$. As in [1] p.1015, we can pass to the limit in (2.3) to obtain some function $U \in C^{2}\left(\mathbb{R}^{2}\right)$ such that $\tilde{u}_{p} \rightarrow U$ as $p \rightarrow \infty$ in $C_{l o c}^{2}\left(\mathbb{R}^{2}\right)$. Passing to the limit in (2.3), we see that $U$ satisfies

$$
-\Delta U=e^{U} \quad \text { in } \mathbb{R}^{2}, \quad \max _{y \in \mathbb{R}^{2}} U(y)=U(0)=0 .
$$

Moreover, by the assumption (1.2) and Fatou's lemma, we can check that

$$
\int_{\mathbb{R}^{2}} e^{U} d y<+\infty
$$

In fact, since $\tilde{u}_{p}(y) \rightarrow U(y)$ a.e. $y \in \mathbb{R}^{2}$, we see

$$
p \log \left(1+\frac{\tilde{u}_{p}}{p}(y)\right) \rightarrow U(y) \quad \text { a.e.y } \in \mathbb{R}^{2}
$$

and

$$
e^{p \log \left(1+\frac{\tilde{u}_{p}}{p}(y)\right)} \rightarrow e^{U(y)} \quad \text { a.e. } y \in \mathbb{R}^{2} .
$$

Thus Fatou's lemma and a simple change of variables using (2.2) imply that

$$
\begin{aligned}
\int_{\mathbb{R}^{2}} e^{U} d y & \leq \liminf _{p \rightarrow \infty} \int_{\Omega_{p}^{1}}\left(1+\frac{\tilde{u}_{p}}{p}(y)\right)^{p} d y \\
& =\liminf _{p \rightarrow \infty} \int_{\Omega}\left(\frac{u_{p}(x)}{\left\|u_{p}\right\|_{\infty}}\right)^{p} p\left\|u_{p}\right\|_{\infty}^{p-1} d x \leq(1 / C) \liminf _{p \rightarrow \infty} p \int_{\Omega} u_{p}^{p} d x
\end{aligned}
$$

where we have used the fact $\left\|u_{p}\right\|_{\infty} \geq C>0$ uniformly for $p$ large. The last term is bounded as before thanks to (1.2), so we get (2.4).

At this point, we see by a result of Chen and Li [2] that

$$
U(y)=-2 \log \left(1+\frac{|y|^{2}}{8}\right)
$$

Next, we define two elliptic operators

$$
\begin{aligned}
L_{p} & :=-\Delta_{x}-p u_{p}^{p-1}(x) \cdot: H_{0}^{1}(\Omega) \rightarrow H^{-1}(\Omega), \\
\tilde{L}_{p} & :=-\Delta_{y}-\left(1+\frac{\tilde{u}_{p}}{p}(y)\right)^{p-1} \cdot: H_{0}^{1}\left(\Omega_{p}^{1}\right) \rightarrow H^{-1}\left(\Omega_{p}^{1}\right) .
\end{aligned}
$$


Note that the operators (2.6) and (2.7) are related to each other by a simple scaling

$$
\left.\varepsilon_{p}^{2} L_{p}\right|_{u_{p}(x)=\left\|u_{p}\right\|_{\infty}\left(1+\frac{\tilde{u}_{p}}{p}(y)\right)}=\tilde{L}_{p}
$$

here, $x=\varepsilon_{p} y+x_{p}^{1}$ for $x \in \Omega$ and $y \in \Omega_{p}^{1}$. Further if we write the $j$-th eigenvalue of an elliptic operator $L$ acting on $H_{0}^{1}(D)$ for a bounded domain $D$ as $\lambda_{j}(L, D), j \in \mathbb{N}$, then

$$
\varepsilon_{p}^{2} \lambda_{j}\left(L_{p}, D\right)=\lambda_{j}\left(\tilde{L}_{p}, D_{p}\right), \quad D_{p}=\frac{D-x_{p}^{1}}{\varepsilon_{p}} .
$$

Now, we claim that there exists $R>0$ such that $\lambda_{1}\left(L_{p}, B\left(x_{p}^{1}, \varepsilon_{p} R\right)\right)<0$ for $p$ sufficiently large.

Indeed, define

$$
w_{p}(y)=y \cdot \nabla \tilde{u}_{p}(y)+\frac{2 p}{p-1}\left(1+\frac{\tilde{u}_{p}}{p}(y)\right), \quad y \in \Omega_{p}^{1} .
$$

Then we have $-\Delta w_{p}(y)=\left(1+\frac{\tilde{u}_{p}(y)}{p}\right)^{p-1} w_{p}(y)$ for $y \in \Omega_{p}^{1}$. Note that $w_{p}(0)=2 p /(p-1) \rightarrow 2$. Also, since $\tilde{u}_{p} \rightarrow U$ in $C_{l o c}^{2}\left(\mathbb{R}^{2}\right)$, we have $w_{p}(y) \rightarrow$ $2\left(8-|y|^{2}\right) /\left(8+|y|^{2}\right)<0$ if $|y|=R>2 \sqrt{2}$ as $p \rightarrow \infty$. Now, set $A_{p}=\{y \in$ $\left.B(0, R): w_{p}(y)>0\right\}, A_{p} \neq \phi$, and

$$
\bar{w}_{p}(y)= \begin{cases}w_{p}(y) & y \in A_{p}, \\ 0 & y \in B(0, R) \backslash \overline{A_{p}} .\end{cases}
$$

Testing

$$
\lambda_{1}\left(\tilde{L}_{p}, B(0, R)\right)=\inf _{v \in H_{0}^{1}(B(0, R))} \frac{\int_{B(0, R)}|\nabla v|^{2} d y-\int_{B(0, R)}\left(1+\frac{\tilde{u}_{p}}{p}(y)\right)^{p-1} v^{2} d y}{\int_{B(0, R)} v^{2} d y}
$$

by $\bar{w}_{p} \in H_{0}^{1}(B(0, R))$, we see that $\lambda_{1}\left(\tilde{L}_{p}, B(0, R)\right) \leq 0$. Strict inequality $\lambda_{1}\left(\tilde{L}_{p}, B(0, R)\right)<0$ actually follows since if equality holds, $\bar{w}_{p}$ would be the first eigenfunction of $\tilde{L}_{p}$ on $B(0, R)$, so it must be strictly positive on $B(0, R)$, which contradicts to the fact that $\bar{w}_{p}$ is 0 near $\partial B(0, R)$. By scaling (2.8), we prove the claim.

If there is another maximum point $x_{p}^{2} \neq x_{p}^{1}$, we repeat the same procedure as before to obtain the ball $B\left(x_{p}^{2}, \varepsilon_{p} R\right)$ such that $\lambda_{1}\left(L_{p}, B\left(x_{p}^{2}, \varepsilon_{p} R\right)\right)<0$. 
We claim that $B\left(x_{p}^{1}, \varepsilon_{p} R\right)$ and $B\left(x_{p}^{2}, \varepsilon_{p} R\right)$ are disjoint for $p$ large. Indeed, since $\tilde{u}_{p} \rightarrow U$ in $C_{l o c}^{2}\left(\mathbb{R}^{2}\right), U(y)=-2 \log \left(1+|y|^{2} / 8\right)$ is strictly concave and $y \cdot \nabla \tilde{u}_{p}(y) \rightarrow y \cdot \nabla U(y)<0$ on $B(0, R) \backslash\{0\}$, we see that $u_{p}$ is also strictly concave and $\left(x-x_{p}^{1}\right) \cdot \nabla u_{p}(x)<0$ on $B\left(x_{p}^{1}, \varepsilon_{p} R\right) \backslash\left\{x_{p}^{1}\right\}$ for $p$ large. The same property holds for $u_{p}$ on $B\left(x_{p}^{2}, \varepsilon_{p} R\right) \backslash\left\{x_{p}^{2}\right\}$, and this concavity property means the claim.

Now, if there are $N$ maximum points $x_{p}^{1}, \cdots, x_{p}^{N}$ of $u_{p}$, we have $N$ open balls $B^{1}, \cdots, B^{N}, B^{j}=B\left(x_{p}^{j}, \varepsilon_{p} R\right)$, which are disjoint, and

$$
\lambda_{1}\left(L_{p}, B^{j}\right)<0 \quad \text { for } j=1, \cdots, N \text {. }
$$

By a variational characterization of $N$-th eigenvalue of $L_{p}$ and the well-known argument as in a proof of Courant's Nodal Domain Theorem, we see that

$$
\lambda_{N}\left(L_{p}, \Omega\right) \leq \sum_{j=1}^{N} \lambda_{1}\left(L_{p}, B^{j}\right) .
$$

From (2.10) and (2.11), we have $\lambda_{N}\left(L_{p}, \Omega\right)<0$. On the other hand, the Morse index of $u_{p}$ is less than or equal to $m$ by assumption, we have $\lambda_{m+1}\left(L_{p}, \Omega\right) \geq$ 0 . Therefore we must have $N \leq m$, and we have proved Theorem 1 .

\section{Proof of Theorem 2.}

Assume $u_{p}$ is a solution of (1.1) of Morse index one with the property (1.2) as $p \rightarrow \infty$. By Theorem 1, we know there exists only one maximum point $x_{p}=x_{p}^{1}$ of $u_{p}$ and on the ball $B=B\left(x_{p}, \varepsilon_{p} R\right) \subset \Omega$,

$$
\lambda_{1}\left(L_{p}, B\right)<0 \quad \text { and }\left(x-x_{p}\right) \cdot \nabla u_{p}(x)<0 \quad\left(\forall x \in B \backslash\left\{x_{p}\right\}\right) .
$$

Now, we claim that $\left(x-x_{p}\right) \cdot \nabla u_{p}(x)<0$ for all $x \in \Omega \backslash \bar{B}$. Indeed, assume there exists some $\bar{x} \in \Omega \backslash B$ such that $\left(\bar{x}-x_{p}\right) \cdot \nabla u_{p}(\bar{x}) \geq 0$. By the variational characterization of the second eigenvalue, we have

$$
\lambda_{2}\left(L_{p}, \Omega\right) \leq \lambda_{1}\left(L_{p}, B\right)+\lambda_{1}\left(L_{p}, \Omega \backslash \bar{B}\right) .
$$

Since $u_{p}$ is a solution of Morse index one, we have $\lambda_{2}\left(L_{p}, \Omega\right) \geq 0$, and thus $\lambda_{1}\left(L_{p}, \Omega \backslash \bar{B}\right)>0$ for $p$ large. On the other hand, by a scaling $\bar{x}=\varepsilon_{p} \bar{y}+x_{p}$ and $\nabla_{y}=\varepsilon_{p} \nabla_{x}$, we have $\bar{y} \cdot \nabla \tilde{u}_{p}(\bar{y}) \geq 0$. By the convexity of $\Omega$, the scaled domain 
$\Omega_{p}=\left(\Omega-x_{p}\right) / \varepsilon_{p}$ is star-shaped with respect to 0 . Hence the Hopf lemma implies that $w_{p}$ in $(2.9)$ satisfies $w_{p}(y)<0$ for $y \in \partial \Omega_{p}$. Since $w_{p}(\bar{y})>0$, there would exist a connected component $C_{p} \subset \Omega_{p} \backslash \bar{B}(0, R), C_{p} \cap \partial \Omega_{p}=\phi$ such that $w_{p}>0$ on $C_{p}$. Then we would have that $\lambda_{1}\left(\tilde{L}_{p}, C_{p}\right) \leq 0$, and by (2.8), $\lambda_{1}\left(L_{p}, \varepsilon_{p}\left(C_{p}+\left\{x_{p}\right\}\right)\right) \leq 0$. Note that $\varepsilon_{p}\left(C_{p}+\left\{x_{p}\right\}\right) \subset \Omega \backslash \bar{B}$. This contradicts to the monotonicity $0<\lambda_{1}\left(L_{p}, \Omega \backslash \bar{B}\right) \leq \lambda_{1}\left(L_{p}, \varepsilon_{p}\left(C_{p}+\left\{x_{p}\right\}\right)\right)$. Thus we have proved $\left(x-x_{p}\right) \cdot \nabla u_{p}(x)<0$ for all $x \in \Omega \backslash\left\{x_{p}\right\}$. The rest of the statement in Theorem 2 is a simple consequence of this inequality.

\section{References}

[1] Adimurthi, and M. Grossi, Asymptotic estimates for a two-dimensional problem with polynomial nonlinearity, Proc. A.M.S. 132(4) (2003) 10131019.

[2] E. Chen, and C. Li, Classification of solutions of some nonlinear elliptic equations, Duke Math. J. 63(3) (1991) 615-622.

[3] K. El Mehdi, and M. Grossi. Asymptotic estimates and qualitative properties of an elliptic problem in dimension two, Advances in Nonlinear Stud. 4(1) (2004) 15-36.

[4] K. El Mehdi, and F. Pacella. Morse index and blow-up points of solutions of some nonlinear problems, Atti. Accad. Naz. Lincei Mat. Appl. 13 (2002) 101-105.

[5] P. Esposito, M. Musso and A. Pistoia. Concentrating solutions for a planar elliptic problem involving nonlinearities with large exponent, Journal of Differential Eq. 227(1) (2006) 29-68.

[6] X. Ren, and J. Wei, On a two-dimensional elliptic problem with large exponent in nonlinearlity, Trans. A.M.S. 343(2) (1994) 749-763.

[7] X. Ren, and J. Wei, Single-point condensation and least-energy solutions, Proc. A.M.S. 124(1) (1996) 111-120. 